\title{
P19 Upper Arm Cuff Inflation Induces Reactive Changes in Systolic Blood Pressure that Could Influence Diagnosis in Some Individuals
}

Louisa Morris*, Dean Picone, Matthew Armstrong, Martin Schultz, James Sharman

Menzies Institute for Medical Research, Hobart, Australia

\begin{abstract}
Introduction: Clinic blood pressure (BP) is performed by inflation of an upper-arm cuff, but this itself may induce a reactive change in BP that could influence diagnosis. This study aimed to determine if a reactive BP change to cuff inflation occurs.

Methods: Cuff BP and beat-to-beat invasive aortic BP were measured simultaneously in 234 individuals $(61 \pm 10$ years, $31 \%$ female) undergoing coronary angiography. Reactive changes in systolic (SBP) and diastolic BP (DBP) were calculated as the difference between invasive aortic BP from resting baseline to immediately post cuff inflation ( $0-10$ seconds). Three groups were defined based on sustained increases $(\geq 5 \mathrm{mmHg})$, decreases $(\leq 5 \mathrm{mmHg})$ or no changes in aortic BP $(<5 \mathrm{mmHg})$.

Results: For aortic SBP, cuff inflation was associated with an increase $(+7.4 \pm 4.8 \mathrm{mmHg})$ in $14 \%(n=33)$ and a decrease $(-7.0 \pm 4.6 \mathrm{mmHg})$ in $21 \%(n=50)$, but no change $(-0.3 \pm 2.6 \mathrm{mmHg})$ in $65 \%(n=151)$ of the population. Cuff SBP and baseline aortic SBP was not different between the three groups $(p>0.05)$, but aortic SBP post-cuff inflation was higher in the group with sustained increases in aortic SBP (ANOVA $p<0.05$ ). Aortic DBP was not significantly changed by cuff inflation. No clinical factors were associated with aortic SBP changes following cuff inflation.

Conclusion: Inflation of an upper-arm cuff induces reactive increases or decreases in SBP in approximately one third of individuals. Whether this may influence diagnosis based on cuff BP needs to be determined.

(C) 2019 Association for Research into Arterial Structure and Physiology. Publishing services by Atlantis Press International B.V. This is an open access article distributed under the CC BY-NC 4.0 license (http://creativecommons.org/licenses/by-nc/4.0/)
\end{abstract}

